\title{
Cisplatin Ineligibility for Patients With Metastatic Urothelial Carcinoma: A Survey of Clinical Practice Perspectives Among US Oncologists
}

\author{
Matthew D. Galsky, ${ }^{\mathrm{a}, *}$ Esprit Ma ${ }^{\mathrm{b}}$, Bijal Shah-Manek ${ }^{\mathrm{c}, 1}$, Rosalina Mills ${ }^{\mathrm{c}}$, Long $\mathrm{Ha}^{\mathrm{c}, 2}$, \\ Craig Krebsbach $^{\mathrm{c}}$, Eric Blouin ${ }^{\mathrm{c}}$, Darren Tayama ${ }^{\mathrm{b}}$ and Sarika Ogale ${ }^{\mathrm{b}}$ \\ ${ }^{a}$ Division of Hematology and Medical Oncology, Icahn School of Medicine at Mount Sinai, Tisch Cancer Institute, \\ New York, NY, USA \\ ${ }^{\mathrm{b}}$ Genentech, Inc, South San Francisco, CA, USA \\ ${ }^{\mathrm{c}}$ Ipsos, San Francisco, CA, USA
}

\begin{abstract}
.
BACKGROUND: Cisplatin-based chemotherapy is the first-line (1L) treatment for eligible patients with locally advanced/metastatic urothelial carcinoma (mUC). However, many patients with $\mathrm{mUC}$ are ineligible for cisplatin-based therapy due to age- or disease-related factors. Specific criteria used by practicing physicians to determine cisplatin ineligibility have not been well characterized.

OBJECTIVE: To understand US oncologists' perspectives and self-reported treatment preferences related to cisplatinineligible patients with $\mathrm{mUC}$.

METHODS: An electronic survey was administered (November-December 2017) to a random sample from a panel of US oncologists who had agreed to participate in periodic surveys. Eligible participants were required to have $\geq 2$ years of clinical experience, have experience treating $\geq 5$ patients with $\mathrm{mUC}$ in the past year, and be board certified and/or eligible. In addition to providing demographic information, clinical preferences, and self-reported practices, participants identified and ranked criteria and reasons for determining cisplatin ineligibility. Descriptive statistics were used to analyze all demographics and responses.

RESULTS: From the 301 respondents, the most commonly identified clinical factors for cisplatin ineligibility were renal dysfunction (78\%) and poor performance status (77\%), followed by neuropathy (47\%), solitary kidney (43\%), hearing loss (43\%), advanced age (43\%), and cardiovascular dysfunction (41\%). Patients were typically deemed ineligible for cisplatin at diagnosis $(58 \%)$ or on initiation of $1 \mathrm{~L}$ metastatic therapy $(61 \%)$. The preferred non-cisplatin $1 \mathrm{~L}$ treatments were checkpoint inhibitors (75\%), followed by carboplatin-based chemotherapy (19\%).

CONCLUSIONS: This survey of US oncologists provides insights into clinical perspectives on cisplatin ineligibility in the context of the evolving treatment landscape for patients with mUC.
\end{abstract}

Keywords: Urothelial carcinoma, survey, cisplatin ineligible, oncologists, chemotherapy, checkpoint inhibitors

\section{INTRODUCTION}

\footnotetext{
${ }^{1}$ Current affiliation: Noesis Healthcare Technologies, Inc. Redwood City, CA, USA.

${ }^{2}$ Current affiliation: Shopify, Montreal, Quebec, Canada.

${ }^{*}$ Correspondence to: Dr. Matthew Galsky, MD, 1190 5th Avenue, New York, NY 10029, USA. Tel.: +1 212241 6756; Fax: +1 212241 7141; E-mail: matthew.galsky@mssn.edu.
}

Urothelial carcinoma is one of the most common cancers, with a worldwide prevalence of more than 1.6 million people and an incidence of 550,000 cases in 2018 [1]. The majority of cases (90-95\%) originate in the lower urinary tract, including the bladder, rather 
than the upper tract [2]. A recent systematic review reported incidence rates of advanced or metastatic bladder cancer to be 3.8, 3.8 and 2.8 per 100,000 in the United States, Europe, and Japan, respectively, with an estimated 5-year survival rate of only 5\% [3].

Historically, cisplatin-based chemotherapy has been the standard first-line (1L) treatment for eligible patients with locally advanced or metastatic urothelial carcinoma (mUC), and although cisplatin-based chemotherapy has demonstrated survival benefit [4], toxicity has been a prominent clinical concern [4-8]. Approximately three-quarters of patients with mUC $(73 \%)$ are 65 years of age or older, more than half of whom are older than 70 years and likely to be ineligible for cisplatin-based therapy $[9,10]$. Overall, advanced age and clinical considerations such as renal function and declining performance status make $30-60 \%$ of patients with mUC ineligible for 1L cisplatin-based chemotherapy [9]. Accordingly, studies of real-world clinical practice and treatment patterns have shown that the likelihood of receiving cisplatin-based therapy decreases with increasing age $[11,12]$.

The recent introduction of immune checkpoint inhibitors for cisplatin-ineligible patients has offered new treatment options and, in turn, brought renewed attention to further characterizing appropriate candidates for cisplatin-based therapy [13]. The antiprogrammed death-ligand 1 (anti-PD-L1) checkpoint inhibitor atezolizumab and anti-programmed death-1 (anti-PD-1) pembrolizumab are approved in the United States, Europe, and elsewhere as $1 \mathrm{~L}$ treatment for cisplatin-ineligible patients with mUC whose tumors express PD-L1 $\geq 5 \%$ (atezolizumab) or who have positive combined scores $\geq 10$ for PD-L1 (pembrolizumab). Atezolizumab and pembrolizumab are also approved for platinum-ineligible patients regardless of PD-L1 expression (in the United States), and for patients with disease progression after platinum therapy regardless of PD-L1 expression, as are nivolumab, avelumab, and durvalumab [14-18].

In the absence of consistent eligibility criteria for drug development trials enrolling cisplatin-ineligible patients with locally advanced or metastatic UC, Galsky and colleagues previously synthesized published trials along with input from an international panel of genitourinary oncologists to identify consensus criteria for cisplatin eligibility [19]. These considerations included renal impairment (creatinine clearance $<60 \mathrm{~mL} / \mathrm{min}$ ), performance status, hearing loss, peripheral neuropathy, and New York Heart
Association Class III heart failure. Although these criteria have proven useful to facilitate drug development for this population with unmet need, the perception of cisplatin ineligibility in real-world clinical practice has not been comprehensively explored. Here, we sought to characterize the perspectives and self-reported preferences of US oncologists regarding management of cisplatin-ineligible patients with locally advanced or metastatic UC.

\section{MATERIALS AND METHODS}

\section{Study design}

We designed and administered an electronic survey to oncologists practicing in the United States. The survey was designed to collect oncologists' demographic information (to evaluate eligibility criteria), clinical preferences, and identification and ranking of their criteria and reasons for determining which patients were ineligible for $1 \mathrm{~L}$ cisplatin treatment of mUC. A copy of the survey is provided in the Supplementary Materials. The survey was distributed between November 21 and December 11, 2017. Participants' contact information was kept separate and blinded from response collection and analysis. This study was exempt from IRB review (Pearl Pathways protocol number 17-062439, https://www.pearlpathways.com), but participants' informed consent was obtained.

Potential participants were identified from a random online panel of about 12,000 US oncologists who had previously elected to participate in anonymized, periodic survey research with research agency Medefield (Medefield.com). All invited oncologists from this trusted partner panel were verified practicing physicians. For eligibility in this survey, participants had to be boardcertified or board-eligible genitourinary medical oncologists, urologic oncologists, or hematologist/oncologists/general medical oncologists; have $\geq 2$ years (and $<35$ years) of clinical experience; and have treated $\geq 5$ patients with mUC in the past year, with $\geq 2$ of these patients having received therapy other than cisplatin.

\section{Statistical analysis}

Descriptive statistics were used to determine the number and characteristics of responders and to synthesize their responses to the survey questions. 
Table 1

Demographic and practice characteristics of survey respondents

\begin{tabular}{|c|c|}
\hline Characteristic, n (\%) unless noted & $\begin{array}{c}\text { Responders } \\
(\mathrm{N}=301)\end{array}$ \\
\hline \multicolumn{2}{|l|}{$\overline{\operatorname{Sex}}$} \\
\hline Male & $246(82)$ \\
\hline Female & $52(17)$ \\
\hline Other & $3(1)$ \\
\hline \multicolumn{2}{|l|}{ Age, years } \\
\hline $31-40$ & $64(21)$ \\
\hline $41-50$ & $126(42)$ \\
\hline $51-60$ & $70(23)$ \\
\hline $61-70$ & $35(12)$ \\
\hline$\geq 71$ & $3(1)$ \\
\hline Unnknown & $3(1)$ \\
\hline \multicolumn{2}{|l|}{ Race/ethnicity } \\
\hline Non-Hispanic white & $152(50)$ \\
\hline Asian & $66(22)$ \\
\hline Black or African-American & $11(4)$ \\
\hline Hispanic & $8(3)$ \\
\hline Mixed race/ethnicity & $16(5)$ \\
\hline Unknown & $48(16)$ \\
\hline \multicolumn{2}{|l|}{ Geographic region } \\
\hline South & $106(35)$ \\
\hline Northeast & $70(23)$ \\
\hline West & $64(21)$ \\
\hline Midwest & $60(20)$ \\
\hline \multicolumn{2}{|l|}{ Specialty } \\
\hline Hematologist/oncologist/general medical oncologist & $246(82)$ \\
\hline Genitourinary oncologist & $36(12)$ \\
\hline Urologic oncologist & $19(6)$ \\
\hline \multicolumn{2}{|l|}{ Primary practice setting } \\
\hline Suburban & $140(47)$ \\
\hline Urban & $133(44)$ \\
\hline Rural & $28(9)$ \\
\hline \multicolumn{2}{|l|}{ Type of practice } \\
\hline Private group practice & $141(47)$ \\
\hline Academic center, hospital/medical center & $108(36)$ \\
\hline Comprehensive cancer center & $36(12)$ \\
\hline Standalone private practice & $11(4)$ \\
\hline Integrated delivery network & $3(1)$ \\
\hline Veterans Health Administration & $2(1)$ \\
\hline Years in practice, mean (SD) & $15(7.0)$ \\
\hline \multicolumn{2}{|l|}{ Patients with mUC treated in the past year } \\
\hline Mean (SD) & $62(79.0)$ \\
\hline Median (IQR) & $35(16-75)$ \\
\hline Patients who received non-cisplatin $1 \mathrm{~L}$ therapy, mean (SD) & $29(46.0)$ \\
\hline
\end{tabular}

\section{RESULTS}

The survey was sent to approximately $12,000 \mathrm{US}$ oncologists, of whom 395 (3\%) were eligible to participate. Among the 395 eligible participants, 301 $(76 \%)$ responded. The majority of respondents were male $(82 \%)$, nearly half $(42 \%)$ were $41-50$ years of age, and most were hematologist/oncologists/general medical oncologists (82\%). Respondents had been in practice for an average of 15 years and on average cared for $\geq 60 \mathrm{mUC}$ patients in the past year (median, 35; IQR, 16-75; Table 1).

Respondents indicated that cisplatin-based chemotherapy was the most common treatment given in the neoadjuvant/adjuvant setting to patients prior to $1 \mathrm{~L}$ treatment of mUC (median percent of patients receiving cisplatin-based chemotherapy, $65 \%$ ), followed by "other chemotherapy" (median, 20\%; Fig. 1A). Cisplatin-based chemotherapy was also cited as the most common treatment for patients 
A. Commonly administered treatments
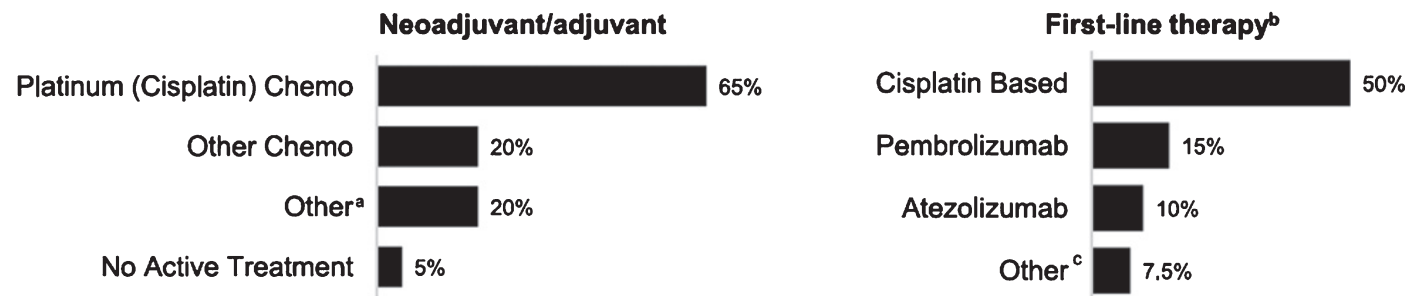

B. Top 3 ranked non-cisplatin first-line therapies

$\nabla$ Ranked 1-3

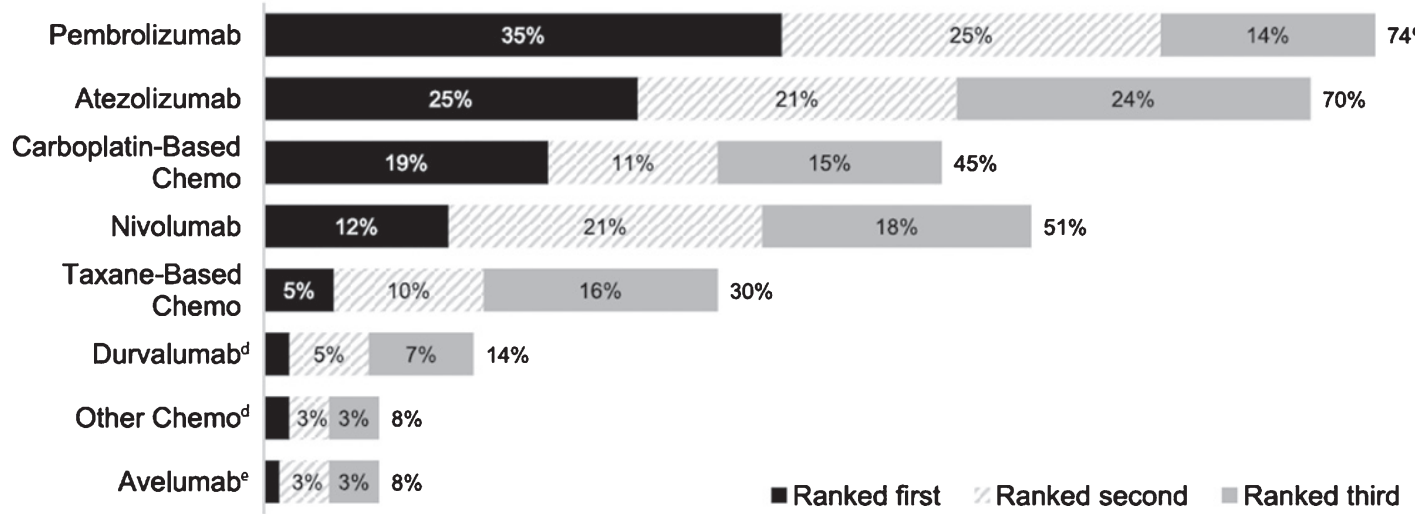

Fig. 1. Reported treatment patterns $(n=301)$. (A) Commonly administered treatments (median percentage of patients receiving treatment in indicated setting). (B). Top 3 ranked non-cisplatin first-line therapies (percentage of respondents). Chemo, chemotherapy. ${ }^{a}$ Cancer immunotherapy/immunotherapy $(n=9)$ and Bacillus Calmette-Guérin, carboplatin, clinical trial, radiotherapy, sparing $(n=1$ each). ${ }^{\mathrm{b}}$ Nivolumab and other chemo (less than $1 \%$ each) not plotted. ${ }^{\mathrm{c}}$ Avelumab and clinical trial $\left(n=1\right.$ each). ${ }^{\mathrm{d}}$ Ranked first by $2 \%$. ${ }^{\mathrm{e}}$ Ranked first by $1 \%$.

treated in the $1 \mathrm{~L}$ setting (median, 50\%), followed by anti-PD-L1/PD-1 checkpoint inhibitors (median, $25 \%$ ). For patients who had already received cisplatin therapy in the neoadjuvant/adjuvant setting, most respondents $(60 \%)$ reported prescribing cisplatin therapy again if patients progressed $\geq 12$ months after neoadjuvant or adjuvant therapy (30\% would prescribe to patients with progression after $\geq 6$ months; $18 \%$ would not prescribe).

The most common clinical factors for considering patients ineligible for cisplatin therapy were renal dysfunction and poor performance status (Fig. 2). The most frequently cited creatinine clearance thresholds for determining cisplatin ineligibility based on renal dysfunction (Table 2) were $<50 \mathrm{~mL} / \mathrm{min}$ $(28 \%),<60 \mathrm{~mL} / \mathrm{min}(16 \%),<40 \mathrm{~mL} / \mathrm{min}(16 \%)$, and $<45 \mathrm{~mL} / \mathrm{min}$ (14\%). Most participants $(67 \%)$ reported using the Cockcroft-Gault equation to determine creatinine clearance, but other methods (not mutually exclusive) were also cited: Chronic Kidney Disease Epidemiology Collaboration (CKD-EPI) by $29 \%$ of respondents, Modification of Diet in Renal Disease (MDRD) equation by $28 \%$, Jelliffe equa- tion by $9 \%$, and other by $0.44 \%$. A total of $55 \%$ of respondents did not differentiate between the terms "cisplatin ineligible" and "cisplatin unfit," and when asked which criteria were used to determine performance status for cisplatin-unfit patients, $77 \%$ of respondents indicated Eastern Cooperative Oncology Group performance status (ECOG PS), 39\% indicated Karnofsky, and 30\% indicated World Health Organization criteria. ECOG PS $\geq 2$ was cited as the threshold for cisplatin ineligibility by the vast majority of respondents (ECOG PS $\geq 1,7 \%$; ECOG PS $2,41 \%$; ECOG PS $>2,52 \%$ ). Additional considerations for cisplatin eligibility included neuropathy (considered always or frequently by $47 \%$ ), having a solitary kidney (43\%), hearing loss (43\%), advanced age $(43 \%)$, and cardiovascular dysfunction $(41 \%)$. Of the 289 respondents who indicated considering age (either seldomly, sometimes, frequently, or always), $40 \%$ indicated $75+$ years as the age group used to define cisplatin ineligibility, 26\% indicated $80+$ years, $24 \%$ indicated $70+$ years, $8 \%$ indicated $65+$ years, and $2 \%$ indicated other. Some physicians also cited diabetes (20\%) or hypertension (19\%) 


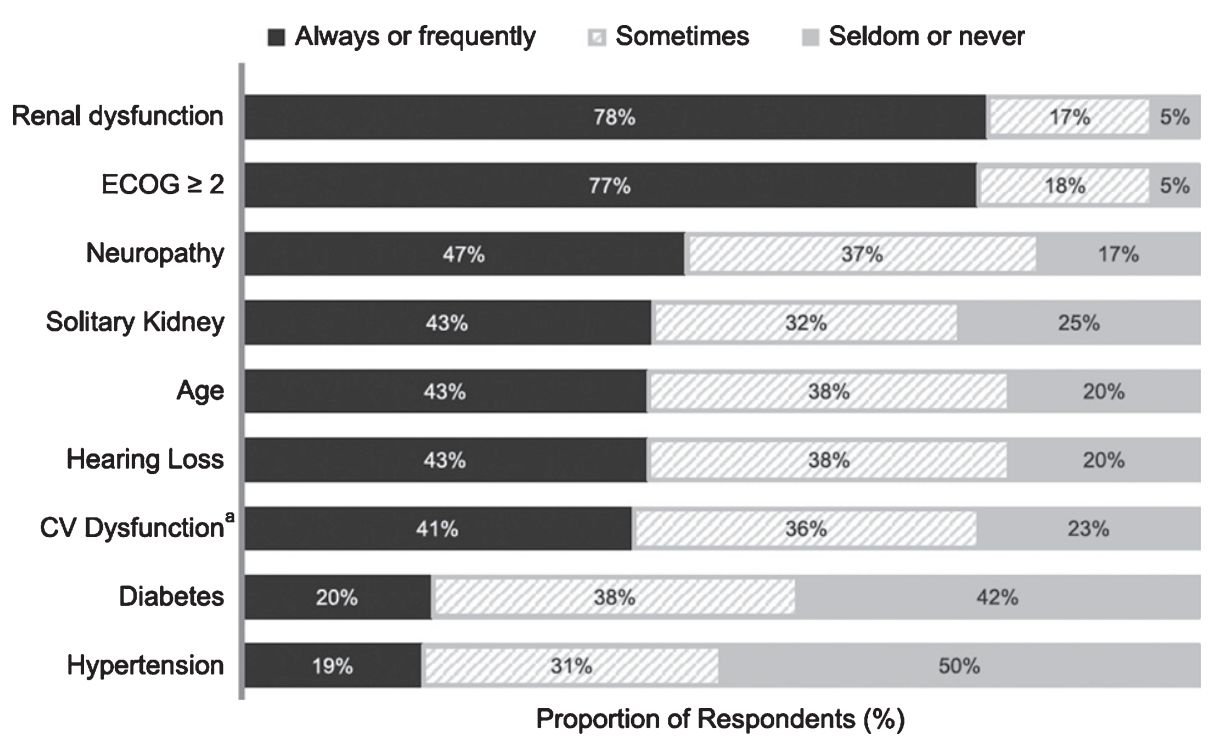

Fig. 2. Most common factors considered to determine cisplatin ineligibility $(n=301)$. ECOG, Eastern Cooperative Oncology Group; CV, cardiovascular. "Other" comorbidities were cited by 10 respondents, without detail related to identification of the comorbid conditions. ${ }^{a}$ Note: CV dysfunction category reflects both heart failure and cardiovascular function.

Table 2

Creatinine clearance threshold used to define cisplatin ineligibility

\begin{tabular}{lc}
\hline $\begin{array}{l}\text { Threshold, } \\
\mathrm{mL} / \mathrm{min}\end{array}$ & $\begin{array}{c}\text { Respondents, } \\
\%\end{array}$ \\
\hline $0-50$ & 28 \\
$0-60$ & 16 \\
$0-40$ & 16 \\
$0-45$ & 14 \\
$0-55$ & 9 \\
$0-30$ & 9 \\
$0-35$ & 8 \\
\hline Other & 0.34 \\
\hline
\end{tabular}

as contributing factors to the treatment decision (Fig. 2).

Oncologists also reported not using $1 \mathrm{~L}$ cisplatin therapy due to prior progression with cisplatin in the neoadjuvant/adjuvant setting $(68 \%)$, patient concern for adverse events (39\%), and patient preference (34\%). In terms of timing, most respondents reported that patients with mUC were typically deemed ineligible for cisplatin at initiation of $1 \mathrm{~L}$ metastatic therapy $(61 \%)$ or diagnosis of metastatic disease (58\%; other periods indicated in Fig. 3).

Most physicians reported that their preferred non-cisplatin $1 \mathrm{~L}$ treatments overall were immune checkpoint inhibitors (top ranked response by $75 \%$ of respondents) followed by carboplatin-based chemotherapy (19\%), citing reasons related to clini-

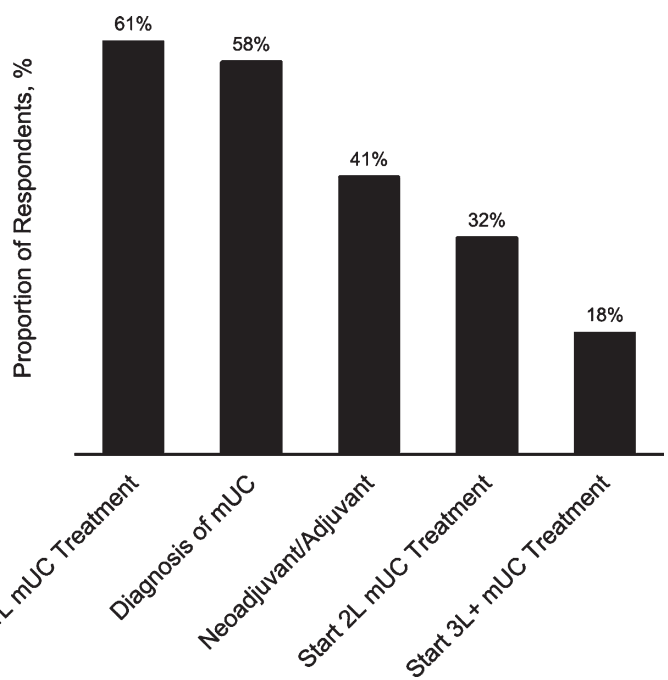

Fig. 3. Typical timing for determination of cisplatin ineligibility $(n=301) .1 \mathrm{~L}$, first-line; $2 \mathrm{~L}$, second-line; $3 \mathrm{~L}+$, third-line or greater; mUC, metastatic urothelial cancer. Response options were not mutually exclusive.

cal efficacy, experience with the preferred treatment, and safety (Fig. 1B). Self-reported adherence to treatment guidelines was relatively high ("Frequently" 67\%; "Sometimes" 23\%), with NCCN (84\%) and ASCO (52\%) cited as non-mutually exclusive reference guidelines. 


\section{DISCUSSION}

This survey of US oncologists provides insights into clinical perspectives on cisplatin ineligibility in the context of the evolving treatment landscape for patients with locally advanced or metastatic UC. In this large survey conducted in 2017, US oncologists treating mUC cited renal impairment and performance status as clear priorities in determining patients' eligibility for cisplatin-based chemotherapy. As expected, respondents also weighed concerns about comorbidities and additional considerations related to individual patients, shared decisionmaking, and clinical judgment. Clinicians reported determining cisplatin eligibility most often at the time of diagnosis or on initiation of $1 \mathrm{~L}$ treatment. Respondents also indicated a strong preference for checkpoint inhibitors over carboplatin-based therapy for non-cisplatin-treated patients in this setting.

This study was conducted to understand real-world practice preferences and decision factors among US oncologists in the modern treatment landscape. Notably, the results of this survey were consistent with those of the previous work by Galsky and colleagues that was done to bring consistency to clinical trial eligibility criteria $[19,20]$. The criteria for patient participation in clinical trials are known to be very different from those underlying real-world treatment decisions, which necessarily require greater flexibility in the risk-benefit calculation as part of a shared decision-making process between patients and providers. In the clinical setting, for example, cisplatin-based therapy may be given in the neoadjuvant setting despite a patient having a certain level of renal impairment, with the intention to achieve a curative outcome. Some of the similarities and differences between this study and the prior survey addressing clinical trial criteria are notable. Both efforts identified renal function and ECOG PS $(\geq 2)$ as the criteria most commonly considered for $1 \mathrm{~L}$ cisplatin ineligibility. Having a solitary kidney was a more prominent factor in this survey, while heart failure and hearing loss seemed relatively consistent factors $[19,20]$.

Results from this survey should be interpreted in light of certain strengths and limitations. This study included a large sample size across a variety of demographic and practice settings in the US, which is important when investigating real-world practices. Although we surveyed oncologists across the country, we did not recruit all practicing oncologists treating patients with mUC, and so these findings are not necessarily representative of overall US practices. Those who opted to participate in the survey may have been different than those who did not participate, leading to the possibility of an undetectable selection bias in the sample. It should be noted that selfreported information may also be subject to potential bias. This survey included only US-based clinicians, and these findings should not be extrapolated to settings outside the United States. This survey investigated treatment decision factors in the context of the available checkpoint inhibitor therapies, which have substantially impacted the treatment landscape for patients with mUC. However, this survey was administered in late 2017, before the 2018 US Food and Drug Administration's update to prescribing information for atezolizumab and pembrolizumab that incorporated PD-L1 status for $1 \mathrm{~L}$ cisplatin-ineligible patients. Responses related to $1 \mathrm{~L}$ treatment preferences for this population might have varied had the survey been conducted after the product label changes. Although our findings are similar to prior work $[19,20]$, this study adds additional insight in terms of the thresholds considered by a larger panel of oncologists (eg, creatinine clearance, definitions of performance status, advanced age).

This study sheds light on the most frequent reasons that patients with $\mathrm{mUC}$ are considered ineligible for cisplatin-based therapy in clinical practice in the United States, extending beyond those established for clinical trial eligibility criteria. Future research may take advantage of advanced observational research platforms and methods to validate or update these findings and to further characterize clinical practice patterns across a larger sample of providers and patients.

\section{AUTHOR CONTRIBUTIONS}

All authors contributed to the conception of the study. All authors contributed to the interpretation of data and preparation of the manuscript, gave approval to submit the manuscript and agree to be accountable for the work.

\section{ETHICAL CONSIDERATIONS}

The study was conducted in accordance with the ethical principles that have their origin in the current Declaration of Helsinki and were consistent with Good Practices for Outcomes Research (International Society of Pharmacoeconomics and Outcomes Research), code of standards and ethics for survey 
research, and other applicable local laws and regulatory requirements.

\section{ACKNOWLEDGMENTS}

Medical writing assistance for this manuscript was provided by Jeff Frimpter, MPH, of Health Interactions, Inc, and funded by F. Hoffmann-La Roche, Ltd.

\section{FUNDING}

F. Hoffmann-La Roche, Ltd. sponsored the study and was involved in study design, analysis and interpretation of results, and development and submission of this report.

\section{CONFLICT OF INTEREST}

M. Galsky has received research support and personal fees (advisory board) from Genentech, Merck, AstraZeneca, Bristol-Myers Squibb, Janssen, and Dendreon, as well as personal fees (advisory board) from BioMotiv, GlaxoSmithKline, Lilly, Astellas, Novartis, Pfizer, EMD-Serono, Seattle Genetics, Incyte, Aileron, Dracen, Inovio, and Dragonfly. E. Ma, D. Tayama, and S. Ogale: Employed by Genentech, Inc, and own Roche stock. B. Shah-Manek, L. Ha, C. Krebsbach, R. Mills, E. Blouin: Employed by Ipsos at the time of analysis. All authors report funding of editorial support from F. Hoffmann-La Roche, Ltd.

\section{SUPPLEMENTARY MATERIAL}

An abbreviated copy of the survey is available as a supplement to this article.

The supplementary material is available in the electronic version of this article: http://dx.doi. org/10.3233/BLC-190235

\section{REFERENCES}

[1] International Agency for Research on Cancer. Global Cancer Observatory. Available from: https://gco.iarc.fr/today/online-analysis-table.

[2] Rouprêt M, Babjuk M, Compérat E, Zigeuner R, Sylvester RJ, Burger M, et al. European Association of Urology Guidelines on upper urinary tract urothelial cell carcinoma: 2015 update. Eur Urol. 2015;68(5): 868-79.

[3] Bharmal M, Guenther S, Kearney M, Kempel-Waibel A. Epidemiology of locally advanced or metastatic urothe- lial cancer in the US, Europe and Japan. Value Health. 2017;20(9):A419.

[4] Loehrer PJ Sr, Einhorn LH, Elson PJ, Crawford ED, Kuebler P, Tannock I, et al. A randomized comparison of cisplatin alone or in combination with methotrexate, vinblastine, and doxorubicin in patients with metastatic urothelial carcinoma: A cooperative group study. J Clin Oncol. 1992;10(7):1066-73.

[5] De Santis M, Bellmunt J, Mead G, Kerst JM, Leahy M, Maroto P, et al. Randomized phase II/III trial assessing gemcitabine/carboplatin and methotrexate/carboplatin/vinblastine in patients with advanced urothelial cancer who are unfit for cisplatin-based chemotherapy: EORTC Study 30986. J Clin Oncol. 2012;30(2):191-9.

[6] Sternberg CN, de Mulder PHM, Schornagel JH, Théodore C, Fossa SD, van Oosterom AT, et al. Randomized phase III trial of high-dose-intensity methotrexate, vinblastine, doxorubicin, and cisplatin (MVAC) chemotherapy and recombinant human granulocyte colony-stimulating factor versus classic MVAC in advanced urothelial tract tumors: European Organization for Research and Treatment of Cancer protocol no. 30924. J Clin Oncol. 2001;19(10):2638-46.

[7] Gabrilove JL, Jakubowski A, Scher H, Sternberg C, Wong G, Groud J, et al. Effect of granulocyte colony-stimulating factor on neutropenia and associated morbidity due to chemotherapy for transitional-cell carcinoma of the urothelium. New Engl J Med. 1988;318(22):1414-22.

[8] von der Maase H, Hansen SW, Roberts JT, Dogliotti L, Oliver T, Moore MJ, et al. Gemcitabine and cisplatin versus methotrexate, vinblastine, doxorubicin, and cisplatin in advanced or metastatic bladder cancer: Results of a large, randomized, multinational, multicenter, phase III study. J Clin Oncol. 2000;18(17):3068-77.

[9] Dash A, Galsky MD, Vickers AJ, Serio AM, Koppie TM, Dalbagni G, Bochner BH. Impact of renal impairment on eligibility for adjuvant cisplatin-based chemotherapy in patients with urothelial carcinoma of the bladder. Cancer. 2006;107:506-13.

[10] SEER. Cancer statistics review (CSR) 1975-2014. Age distribution at diagnosis. https://seer.cancer.gov/ archive/csr/1975_2014/results_merged/topic_age_dist.pdf. Accessed January 3, 2019.

[11] Galsky MD, Pal SK, Lin S-W, Ogale S, Zivkovic M, Simpson J, et al. Real-world effectiveness of chemotherapy in elderly patients with metastatic bladder cancer in the United States. Bladder Cancer. 2018;4:227-38.

[12] Sonpavde G, Watson D, Tourtellott M, Cowey CL, Hellerstedt B, Hutson TE, et al. Administration of cisplatin-based chemotherapy for advanced urothelial carcinoma in the community. Clin Genitourin Cancer. 2012;10(1):1-5.

[13] Balar AV, Galsky MD, Rosenberg JE, Powles T, Petrylak DP, Bellmunt J, et al. Atezolizumab as first-line treatment in cisplatin-ineligible patients with locally advanced and metastatic urothelial patients with locally advanced and metastatic urothelial carcinoma: A single-arm, multicenter, phase 2 trial. Lancet. 2017;389:67-76.

[14] Tecentriq (atezolizumab) [package insert]. South San Francisco, CA: Genentech, Inc; 2019.

[15] Keytruda (pembrolizumab) [package insert]. Whitehouse Station, NJ: Merck \& Co., Inc; 2019.

[16] Opdivo (nivolumab) [package insert]. Princeton, NJ: Bristol-Myers Squibb Company; 2019.

[17] Bavencio (avelumab) [package insert]. Rockland, MA: EMD Serono, Inc; 2018. 
[18] Imfinzi (durvalumab) [package insert]. Wilmington, DE: AstraZeneca Pharmaceuticals LP; 2018.

[19] Galsky MD, Hahn NM, Rosenberg J, Sonpavde G, Hutson $\mathrm{T}$, Oh WK, et al. A consensus definition of patients with metastatic urothelial carcinoma who are unfit for cisplatinbased chemotherapy. Lancet Oncol. 2011;12:211-4.
[20] Galsky MD, Hahn NM, Rosenberg J, Sonpavde G, Hutson T, Oh WK, et al. Treatment of patients with metastatic urothelial cancer "unfit" for cisplatin-based chemotherapy. J Clin Oncol. 2011;29(17):2432-8. 